\title{
Terapia de juicio de lateralidad e imaginería de movimiento y ejercicios de activación muscular selectiva glenohumerales en sujetos con ruptura masiva del manguito rotador: serie de casos
}

\author{
D. Rubio Oyarzún ${ }^{1,2}$, F. Araya Quintanilla ${ }^{1,3}$, H. Gutiérrez Espinoza ${ }^{1,4}$, C. Olguín Huerta ${ }^{1}$, Y. Fritz ${ }^{1,2}$ \\ y L. Arias Poblete 5 \\ ${ }^{\text {I}}$ Escuela de Kinesiología. Facultad de Ciencias de la Salud. Universidad de Las Américas. ${ }^{2}$ Clínicas \\ Quiroprácticas Raquis. ${ }^{3}$ Escuela de Kinesiología. Facultad de Ciencias de la Salud. Universidad Gabriela \\ Mistral. ${ }^{4}$ Hospital Clínico San Borja Arriarán. ${ }^{5}$ Escuela de Kinesiología. Facultad de Ciencias de la \\ Rehabilitación. Universidad Andrés Bello. Santiago. Chile
}

Rubio Oyarzún D, Araya Quintanilla F, Gutiérrez Espinoza H, Olguín Huerta C, Fritz Y y Arias Poblete L. Terapia de juicio de lateralidad e imaginería de movimiento y ejercicios de activación muscular selectiva glenohumerales en sujetos con ruptura masiva del manguito rotador: serie de casos. Rev Soc Esp Dolor 2018;25(4):197-206.

\begin{abstract}
Introduction: Massive tear of the rotator cuff (DMMR) is a degenerative clinical condition, which corresponds to a $5 \mathrm{~cm}$ rupture, or one that compromises two or more tendons of the rotator cuff (MR), generating loss of functionality and disabling pain.

Objective: To describe changes in pain and shoulder function following a 6-week program of lateral-imaging and motion imaging therapy and selective glenohumeral activation exercises in subjects with massive rotator cuff tears.

Methods: This study is a descriptive research and design of a series of cases, with a sample of 50 participants with diagnosis of rotator cuff mastication. Patients underwent a glenohumeral selective exercise program plus laterality and motion imaging therapy for 6 weeks. The variables of function, pain, abduction $\mathrm{ROM}$ and shoulder flexion were measured at sixth week and sixth month of evolution.

Results: There were significant differences in pain intensity, shoulder function, AROM flexion and shoulder abduction AROM, after the intervention ( $p>0.05)$. Statistically significant differences were found for all outcome measures between the intervention and the sixth follow-up month $(p<0.05)$. Only the
\end{abstract}

pain of the statistically significant differences between the sixth week and the sixth month of follow-up $(p=0.01)$.

Conclusion: The application of laterality trial therapy and motion imaging added to a program of selective exercises stabilizadores glenohumerales during 6 weeks, could improve shoulder function, decrease pain and increase AROM flexion and shoulder abduction in patients with massive rupture of the rotator cuff.

Key words: Graduated motor imaging, therapeutic exercise, chronic pain, massive rupture rotator cuff.

\section{RESUMEN}

Introducción: El desgarro masivo del maguito rotador (DMMR) es una condición clínica degenerativa que corresponde a una ruptura de $5 \mathrm{~cm}$, o una que compromete a dos o más tendones del manguito rotador (MR), generando pérdida de funcionalidad y dolor incapacitante.

Objetivo: Describir los cambios en el dolor y función de hombro posterior a un programa de 6 semanas de terapia de juicio de lateralidad e imaginería de movimiento y ejercicios de activación selectiva glenohumerales en sujetos con rotura masiva del manguito rotador.

Métodos: Este estudio es una investigación descriptiva y diseño serie de casos, con una muestra de 50 participantes con diagnóstico de ruptura masiva de manguito rotador. Los pacientes realizaron un programa de ejercicios selectivos glenohumerales más terapia de juicio de lateralidad e imaginería de movimiento durante 6 semanas. Se midieron las variables de función, dolor, $\mathrm{ROM}$ de abducción y flexión de hombro, a la sexta semana y al sexto mes de evolución. 
Resultados: Existen diferencias significativas en la intensidad del dolor, función de hombro, AROM de flexión y AROM de abducción de hombro, posterior a la intervención $(p<0,05)$. Existen diferencias estadísticamente significativas para todas las medidas de resultados entre la intervención y el sexto mes de seguimiento $(p<0,05)$. Solo el dolor presentó diferencias estadísticamente significativas entre la sexta semana y el sexto mes de seguimiento $(p=0,01)$.

Conclusión: La aplicación de la terapia de juicio de lateralidad e imaginería de movimiento adicionada a un programa de ejercicios selectivos estabilizadores glenohumerales durante 6 semanas podría mejorar la función de hombro, disminuir el dolor y aumentar los AROM de flexión y abducción de hombro en pacientes con ruptura masiva del manguito rotador.

Palabras clave: Imaginería motora graduada, ejercicio terapéutico, dolor crónico, ruptura masiva manguito rotador.

\section{INTRODUCCIÓN}

El desgarro masivo del maguito rotador (DMMR) es una condición clínica degenerativa que puede comprometer a los músculos subescapular, supraespinoso, infraespinoso y redondo menor, lo cual corresponde a una ruptura de $5 \mathrm{~cm}$, o una lesión de dos o más tendones del manguito rotador (MR) (1). Se estima que la prevalencia es entre un 10 y un $40 \%$ en la población entre los 50-55 años, llegando a un $60 \%$ en los sujetos mayores de 70 años (2). Su presentación clínica es manifestada por dolor incapacitante, disminución del rango activo de movimiento (AROM) en flexión anterior y rotación externa, impotencia funcional $(3,4)$ y en algunos casos pseudoparálisis (5). Junto a esto, Werner en el año 2005 utilizó el término, definiéndolo como una elevación activa y pasiva del hombro inferior a $90^{\circ}$, causada por un desgarro masivo del manguito rotador. Desde entonces, la literatura ha utilizado el término para abarcar esta restricción de movimiento y que a menudo se asocian a dolor, deterioro degenerativo y pérdida de rotación externa (5). Generalmente estos deterioros son acompañados de un edema óseo e infiltración grasa en el tendón, provocando un ascenso de la cabeza humeral como una de las principales complicaciones médicas (6).

Paralelamente, algunos estudios electromiográficos han reportado una actividad muscular aumentada del músculo dorsal ancho y pectoral mayor $(7,8)$, con el fin de contrarrestar el ascenso de la cabeza humeral e impidiendo un contacto mecánico mayor. Bajo este mismo punto se ha evidenciado una mayor actividad electromiográfica del músculo deltoides medio y anterior $(9,10)$ para facilitar la abducción y elevación del brazo de manera compensatoria. No obstante, todas estas alteraciones biomecánicas y neuromusculares son estrategias neuromotoras adaptativas desarrolladas en el sistema nervioso central (SNC) $(11,12)$, que buscan reprogramar el patrón de activación muscular del manguito rotador y musculatura escapular para compensar este déficit motor y mantener la funcio- nalidad de la extremidad superior $(12,13)$. Sin embargo, estos mismos déficit motores se pueden utilizar para generar una nueva estrategia de reprogramación del movimiento a través de los músculos dorsal ancho, pectoral mayor e infraespinoso, que buscan deprimir la cabeza humeral $(7-9,11)$.

En la actualidad, una estrategia utilizada en la terapia física (TF) para reprogramar al sistema nervioso en patologías de dolor crónico y déficit neuromotores es la imaginería motora graduada (IMG) $(14,15)$, que corresponde a una serie secuencial de ejercicios corticales dirigidos a restablecer los cambios neuroplásticos con la intención de disminuir la experiencia del dolor y restablecer la redistribución de la actividad muscular en disfunción (16,17). Según lo anterior, se ha evidenciado que las zonas del cuerpo están representadas en la corteza a través de una red neuronal (18) y que en pacientes con dolor presentan diferencias en las representaciones topográficas de la corteza somatosensorial, en comparación con sujetos sin dolor (19). Estos cambios se manifiestan en los mapas cerebrales, que se expanden o disminuyen su representación a nivel cortical (20) y se postula que estos cambios conducen al desarrollo, mantenimiento del dolor crónico y la pérdida de funcionalidad $(21,22)$.

La IMG utiliza diversas estrategias de reprogramación cortical y que se divide en tres etapas: primero reconocer partes o movimientos del hemicuerpo izquierdo o derecho (juicio de lateralidad); en segundo lugar visualizar estática o dinámicamente una parte del cuerpo, imaginando los movimientos normales de una articulación, y en tercer lugar se finaliza con la terapia espejo (23-25). En la actualidad, algunos estudios han demostrado que la IMG disminuye el dolor (14,26), mejora la sensibilidad(14), aumenta el movimiento (24) y la función en sujetos con dolor crónico (27). Esta terapia se fundamenta en disminuir la hiperactividad del SNC, permitiendo a los pacientes tolerar terapias activas para normalizar el movimiento y las alteraciones funcionales logrando disminuir el dolor (28). Según lo anterior surge nuestra pregunta: en pacientes con ruptura masiva del manguito rotador, ¿pueden existir cambios en el dolor y función de hombro al adicionar un programa de 6 semanas de imaginería motora graduada a ejercicios de activación selectiva glenohumeral?

\section{OBJETIVO}

Describir los cambios en el dolor y función de hombro al adicionar un programa de 6 semanas de terapia de juicio de lateralidad e imaginería de movimiento a ejercicios de activación selectiva glenohumerales en sujetos con rotura masiva del manguito rotador.

\section{MÉTODOS}

El presente estudio corresponde a un estudio de tipo descriptivo con un diseño serie de casos. 


\section{Muestra}

El estudio se realizó en el laboratorio de kinesiología de Universidad de las Américas ubicada en Manuel Montt 948, Providencia, Santiago, durante el periodo de septiembre de 2016 a abril de 2017. La muestra fue constituida por 50 sujetos, 18 hombres y 32 mujeres, con una edad promedio de 68,3 años, con diagnóstico médico e imagenológico de ruptura masiva del manguito rotador.

La muestra se obtuvo de manera no probabilística, ya que fue por orden de consecución de llegada de los pacientes. Todos los sujetos incluidos en el estudio aceptaron y firmaron el consentimiento informado. Adicionalmente, solo recibían paracetamol de 500 miligramos cada 8 horas en caso de presentar un dolor intolerable, cada dosis fue prescrita por el médico traumatólogo del servicio de atención primaria de salud, al cual el participante acudía para control y tratamiento de su condición clínica, solo 9 pacientes $(18 \%)$ informaron haber consumido el medicamento en las dosis prescritas. Esta prescripción no se considera una co-intervención debido a que las medicaciones de los pacientes eran solo en el caso de requerimiento e indicado por el médico, no así como una co-intervención externa del tratamiento que pudiese generar una fuente potencial de sesgo.

Las características basales se presentan en la Tabla I. La edad de los pacientes varió entre 60 y 75 años, con un promedio de 68,3 años. Respecto a la muestra, el $64 \%$ fueron mujeres y $36 \%$ hombres.

TABLA I

ANÁLISIS DEMOGRÁFICO DE LA MUESTRA EN LA LÍNEA DE BASE

\begin{tabular}{|c|l|}
\hline $\begin{array}{c}\text { Tamaño de la } \\
\text { muestra }\end{array}$ & 50 sujetos: $100 \%$ \\
\hline Género masculino & 8 hombres: $36 \%$ \\
\hline Género femenino & 32 mujeres: $64 \%$ \\
\hline Edad (promedio) & $60-75$ años $(68,3$ años) \\
\hline $\begin{array}{c}\text { Tiempo de evolución } \\
\text { de condición } \\
\text { (Promedio) }\end{array}$ & Tango de 7 a 10 años $(9,4$ años) \\
\hline $\begin{array}{c}\text { Dolor }- \text { EVA } \\
\text { Función - Score de } \\
\text { Constant }\end{array}$ & Promedio 5,5 cm (DE 1,06$)$ \\
\hline $\begin{array}{c}\text { Rango de } \\
\text { movimiento flexión }\end{array}$ & Promedio $70,64^{\circ} \mathrm{DE}\left(15,65^{\circ}\right)$ \\
\hline $\begin{array}{c}\text { Rango de } \\
\text { movimiento } \\
\text { abdución }\end{array}$ & Promedio 57,43 ${ }^{\circ} \mathrm{DE}\left(15,84^{\circ}\right)$ \\
\hline
\end{tabular}

En cuanto a la dominancia, el $94 \%$ eran diestros y el $6 \%$ zurdos, el hombro comprometido estaba en relación con la dominancia del paciente.

Con respecto a las complicaciones asociadas a la intervención, en la segunda semana de tratamiento 3 pacientes informaron dolor más intenso, sin embargo no requirió una visita al médico traumatólogo para evaluar su condición. A los 6 meses de seguimiento ningún paciente informó de complicaciones asociadas con la intervención recibida.

Con respecto a las pérdidas, todos los pacientes completaron el estudio y las evaluaciones en el seguimiento, evitando el sesgo de desgaste.

\section{Criterios de inclusión}

- Sujetos con diagnóstico médico e imagenológico de ruptura masiva del manguito rotador, a través de resonancia nuclear magnética o ecografía.

- Hombres y mujeres igual o mayores a 60 años.

- Sujetos que sean capaces de seguir órdenes simples.

- Sujetos que hayan aceptado y firmado el consentimiento informado.

\section{Criterios de exclusión}

- Sujetos que previamente hayan sido intervenidos quirúrgicamente del manguito rotador.

- Sujetos que hayan recibido tratamiento de corticoides mediante cualquier vía de administración en los últimos 6 meses.

- Sujetos que presenten alteraciones neurológicas centrales o periféricas.

- Sujetos que presenten rotura masiva del manguito rotador por origen traumático agudo como por ejemplo la fractura de humero proximal.

\section{Variables del estudio}

Se han estudiado las siguientes variables:

- Intensidad de dolor.

- Función de hombro.

- Rango de movimiento de flexión de hombro y abducción de hombro.

\section{Material usado para el estudio}

Para la valoración de la intensidad del dolor se utilizó la escala visual análoga (EVA), que consiste en una línea horizontal de $10 \mathrm{~cm}$ de longitud: el extremo izquierdo representa $0 \mathrm{o}$ sin dolor, y el extremo derecho representa $10 \mathrm{o}$ el peor 
dolor imaginable. La distancia en centímetros desde el punto de "no dolor" a la marcada por el paciente representa la intensidad de dolor. Esta evaluación puede disponer o no de marcas entre cada centímetro, aunque para algunos autores pierde precisión (29). Se solicitó a cada paciente que dibujara una línea vertical y que indicara la magnitud del dolor experimentado al momento de la evaluación.

Se recomienda este sencillo y reproducible método de valoración unidimensional para su inclusión en la evaluación de todos los pacientes con patologías de miembro superior (29-32).

La función de hombro se valoró a través del score de Constant-Murley, debido a su confiabilidad en la práctica clínica (30). Tiene un puntaje total de 100 puntos, entregando una correlación positiva: a mayor puntuación, mayor función. Esta escala incluye cuatro parámetros:

- Dolor: donde se utiliza una escala de valoración verbal en la que a la ausencia de dolor se le asignan 15 puntos, un dolor suave 10 puntos, un dolor moderado 5 puntos y un dolor severo 0 puntos.

- Actividades de la vida diaria: esta incluye cuatro categorías y puede alcanzar hasta 20 puntos, donde comienza con la actividad laboral de 0 a 4 puntos, actividad de tiempo libre de 0 a 4 puntos, sueño de 0 a 2 puntos y posición de la mano de 2 a 10 puntos, contando de dos en dos.

- Con respecto al rango de movilidad se valoran cuatro movimientos, elevación anterior de 0 a 10 puntos, elevación lateral de 0 a 10 puntos, rotación externa de 0 a 10 puntos y rotación interna de 0 a 10 puntos, tendiendo solo en consideración el grado de movimiento activo (AROM). Para medir la elevación anterior y lateral, los autores del test recomiendan usar un goniómetro, con el paciente sentado y la espalda apoyada en el respaldo, para compensaciones asociadas a las inclinaciones del tronco (30).

- En relación con la flexión y abducción de hombro activa, se evaluaron mediante goniometría con el paciente en posición sedente, y para permitir la consistencia de las mediciones pre y postintervención se marcaron los reparos óseos anatómicos sobre la piel de cada paciente, ya que esto entrega una buena confiabilidad en evaluaciones goniométricas de hombro $(33,34)$.

Para determinar diferencias estadísticamente significativas, el cambio mínimo detectable para la flexión de hombro ha sido reportado de 8 grados, y la diferencia clínica mínima detectada depende de la patología del paciente (33).

\section{Programa de intervención}

La intervención consistió en un programa kinésico, enfocado en terapia de juicio de lateralidad e imaginería del movimiento y tres ejercicios selectivos estabilizadores glenohumerales durante seis semanas. Los pacientes acudieron a terapia 4 veces por semana con una duración de 60 minutos por sesión. No se les realizó ninguna otra modalidad de intervención fisioterapéutica.

Todas las medidas de resultados fueron registradas antes de la intervención, al final de la intervención ( $6^{\mathrm{a}}$ semana) y a los 6 meses de evolución. Todas las evaluaciones mencionadas las registró un profesional médico traumatólogo externo al estudio, con más de 10 años de experiencia clínica.

\section{Intervención de lateralidad e imaginería del movi- miento}

Con respecto a la IMG, la primera etapa consistió en la restauración de la lateralidad, que es la capacidad de reconocer una parte del cuerpo como perteneciente al hemicuerpo izquierdo o derecho, y para esto se utilizó el software Recognise del grupo Neuro Orthopaedic Institute (NOI) (23). Cada paciente se sentó frente a un computador durante 15 minutos donde se le solicitó identificar rápidamente si las imágenes correspondían a un hombro del lado derecho o izquierdo; estas imágenes aparecen en distintas posiciones y situaciones cotidianas que presentan formas bien definidas o imágenes abstractas (Figura 1).

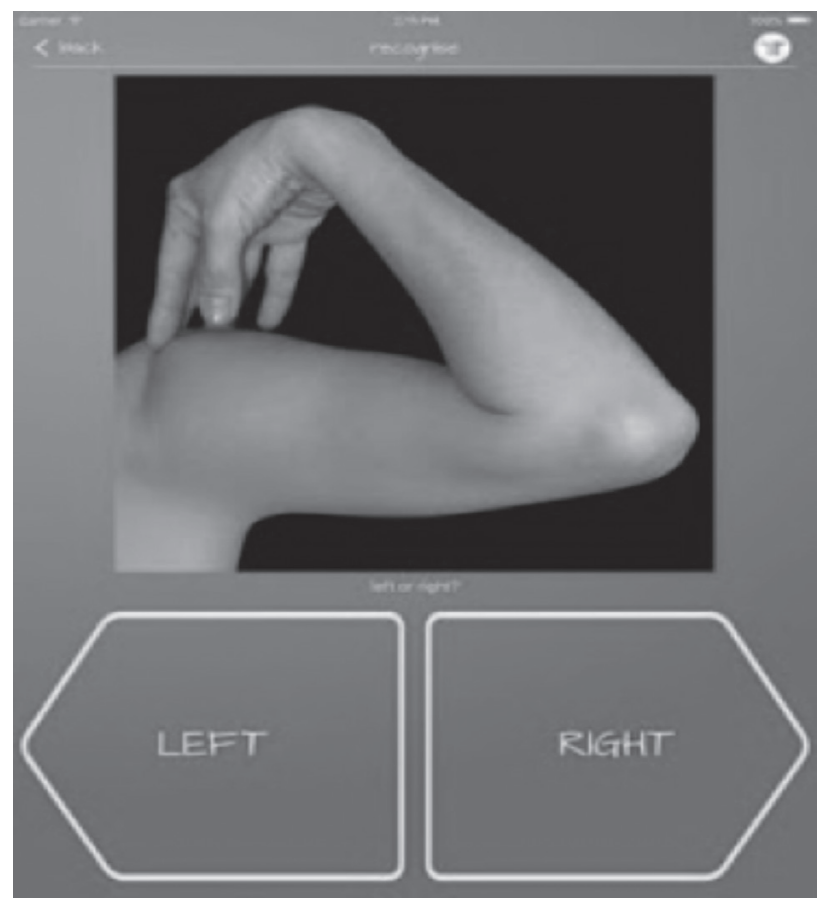

Fig. 1. Imagen de juicio de lateralidad hombro derecho, del software Recognise del grupo Neuro Orthopaedi cInstitute (NOI). 
Durante la segunda etapa, el terapeuta les solicitó imaginar movimientos indoloros del miembro superior afectado por 15 minutos. Primero se indicó que visualizaran los movimientos de hombro que se realizan en los 3 planos de movimientos, comenzando con flexión-extensión, luego abducción-adducción y finalmente con los movimientos de rotaciones, para posteriormente imaginar acciones enfocadas de carácter cotidiano como actividades de la vida diaria, como por ejemplo alcanzar un objeto sobre una repisa, colgar una prenda de vestir (25).

\section{Ejercicios estabilizadores glenohumerales selectivos}

El presente programa de ejercicios selectivos ha sido propuesto en base a los análisis de los estudios electromiográficos de Campbell y cols. (7), Park y cols. (8) y Hansen y cols. (9).

\section{Activación selectiva de depresores humerales $(7,8)$}

Los pacientes se posicionaron en sedente sobre una silla y se les solicitó flexionar los hombros hasta $60^{\circ}$ y el antebrazo flexionado en $130^{\circ}$, para luego apoyar los codos sobre una mesa. Posteriormente se les indicó que debían presionar los codos en dirección al suelo de manera indolora durante 10 segundos, realizándolo 10 veces (Figura 2).

\section{Activación selectiva de deltoides anterior y serrato mayor (9)}

Los pacientes se posicionaron en sedente sobre una silla con apoyabrazos, el brazo estaba en posición neutral y el antebrazo en pronación y flexión de $90^{\circ}$; se les solicitó realizar un empuje anterior con la mano abierta sobre un cojín de manera indolora durante 10 segundos, realizándolo 10 veces (Figura 3 ).

\section{Activación selectiva de deltoides medio (9)}

Los pacientes se posicionaron en sedente sobre una silla y se les solicitó realizar una abducción de hombro en el plano escapular. La posición de inicio fue con el codo en flexión de $70^{\circ}$ a $90^{\circ}$ en posición neutra del antebrazo, y en $45^{\circ}$ de abducción de hombro apoyado sobre una cuña, para luego realizar desde ese rango un movimiento activo de abducción en el plano escapular hasta los $90^{\circ}$, o la máxima abducción posible de manera indolora. Se realizaron 6 series de 10 repeticiones (Figura 4).

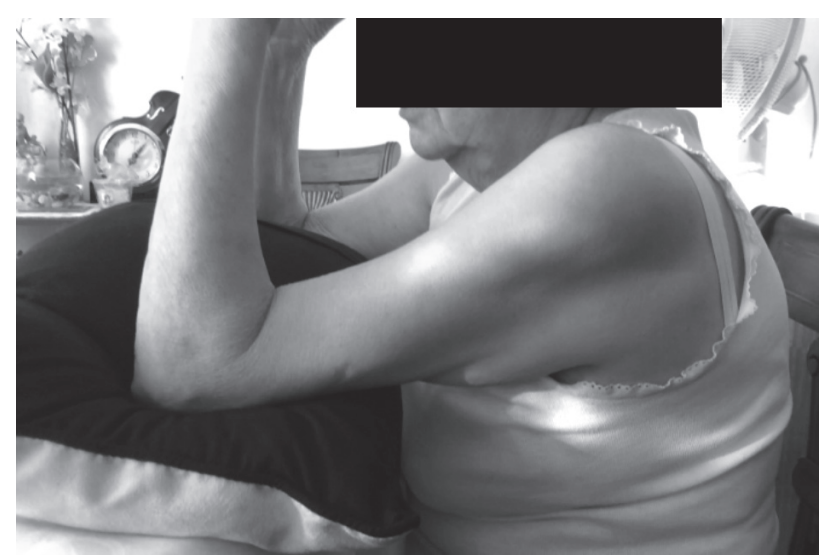

Fig. 2. Ejercicio de activación selectiva de los depresores humerales, dorsal ancho, redondo mayor y pectoral mayor.

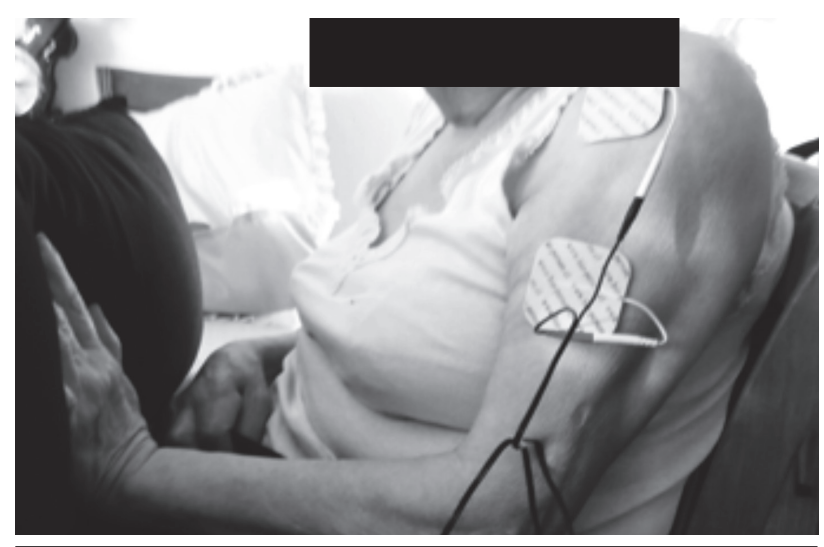

Fig. 3. Ejercicio de activación selectiva del deltoides anterior y serrato mayor.

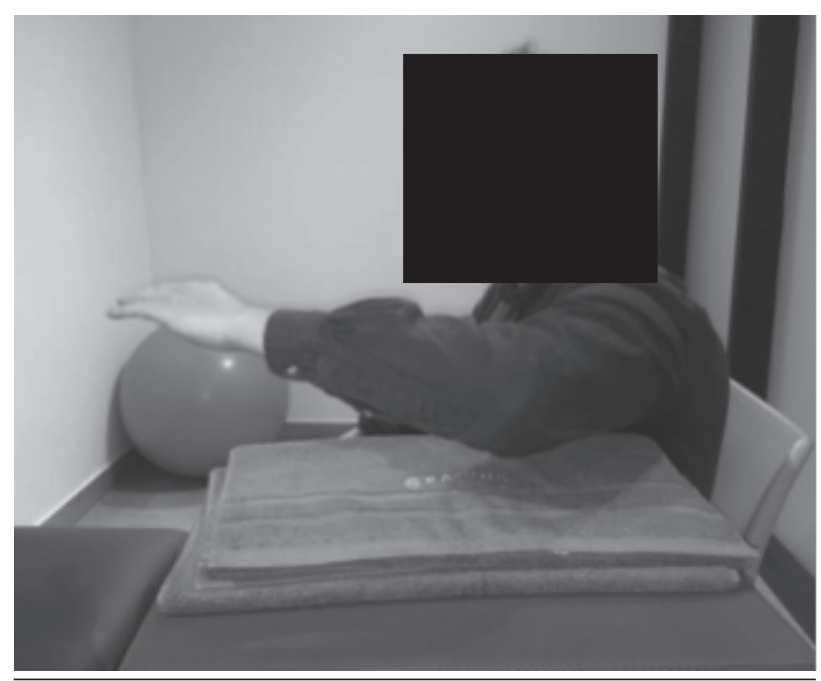

Fig. 4. Ejercicio de activación selectiva del deltoides medio. 


\section{Análisis estadístico}

Los datos se recopilaron y se introdujeron en el software Excel para la tabulación. El análisis estadístico se realizó posteriormente utilizando el software IBM SPSS statistics 32 para Windows. Las variables cuantitativas se presentan como media y desviación estándar, y las variables cualitativas como número y porcentaje. Para determinar las pruebas estadísticas a utilizar, el primer análisis evaluó la distribución normal, utilizando la prueba de Shapiro-Wilk (Tabla II). Se examinaron las diferencias en las puntuaciones del score de Constant total, la EVA y los ROM activos de flexión y abducción de hombro previo al tratamiento, a las 6 semanas y al sexto mes. Debido a que se realizaron 3 evaluaciones, se utilizó ANOVA o prueba de Friedman para muestras dependientes. Se utilizó ANOVA de una vía con medidas repetidas para la diferencia antes del tratamiento, a las 6 semanas y a los seis meses, con el tiempo como variable independiente, para la diferencia específica entre los 3 periodos de evolución se utilizó el test de Bonferroni, estableciendo una significancia estadística de $\mathrm{p}<0,05$.

\section{RESULTADOS}

\section{Dolor}

En la intensidad de dolor, medido con EVA antes de la intervención, fue de $5,5 \mathrm{~cm}$ DE $(1,06)$. A la sexta sema-

TABLA II

ANÁLISIS DE NORMALIDAD

\begin{tabular}{|l|c|}
\hline \multicolumn{2}{|c|}{ Análisis de normalidad } \\
\hline Score de Constant I & $p$-valúe \\
\hline ROM activo de flexión I & $0,01^{*}$ \\
\hline EOM activo de abducción I & $0,18^{*}$ \\
\hline ENA I & $0,31^{*}$ \\
\hline Score de Constant F & $0,31^{*}$ \\
\hline ROM activo de flexión F & 0 \\
\hline ROM activo de abducción F & 0 \\
\hline EVA final & 0,28 \\
\hline Score de Constant S & 0 \\
\hline ROM activo de flexión S & 0 \\
\hline ROM activo de abducción S & 0 \\
\hline EVA S & 0,33 \\
\hline
\end{tabular}

na fue de $1,9 \mathrm{~cm}$ DE $(1,48)$ y al $6^{\circ}$ mes de seguimiento fue de $3,1 \mathrm{~cm} \mathrm{DE}(0,99)$. Existen diferencias estadísticamente significativas en las tres evaluaciones. EVA preintervención versus postintervención $(\mathrm{p}=0,01)$. EVA pre intervención versus al $6^{\circ}$ mes de seguimiento $(\mathrm{p}=0,01)$ y EVA a la sexta semana, versus al $6^{\circ}$ mes de seguimiento $(\mathrm{p}=0,01)$ (Figura 5).

\section{Función}

En la función antes de la intervención el promedio del score de Constant fue de 38,46 puntos DE $(16,32)$. A la sexta semana fue de 63,89 puntos DE $(17,22)$ y al $6^{\circ}$ mes fue de 62,84 puntos DE $(16,88)$. Existen diferencias estadísticamente significativas entre el score de Constant preintervención versus postintervención $(\mathrm{p}=0,01)$, y entre el score de Constant preintervención versus al final del $6^{\circ}$ mes de intervención $(p=0,01)$ (Figura 6). Cabe destacar que, en la valoración de función por Score de constant se extrajo el valor final total de la escala.

\section{AROM de flexión}

En el AROM de flexión antes de la intervención, el promedio fue de $70,64^{\circ} \mathrm{DE}\left(15,65^{\circ}\right)$. A la sexta semana fue de $110,64^{\circ} \mathrm{DE}\left(27,98^{\circ}\right)$ y al $6^{\circ}$ mes fue de $105,51^{\circ}$ DE $\left(24,94^{\circ}\right)$. Existen diferencias estadísticamente significativas entre el AROM de flexión preintervención versus el postintervención $(\mathrm{p}=0,01)$, y además entre el AROM de flexión preintervención versus al final del $6^{\circ}$ mes $(\mathrm{p}=0,02)$ (Figura 7).

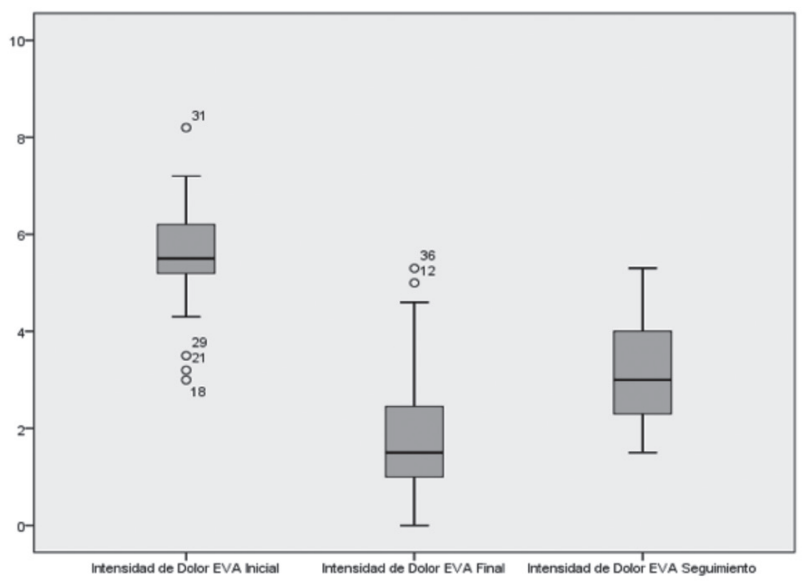

Fig. 5. Puntuación de dolor (EVA), preintervención, a la sexta semana y al sexto mes de seguimiento. 


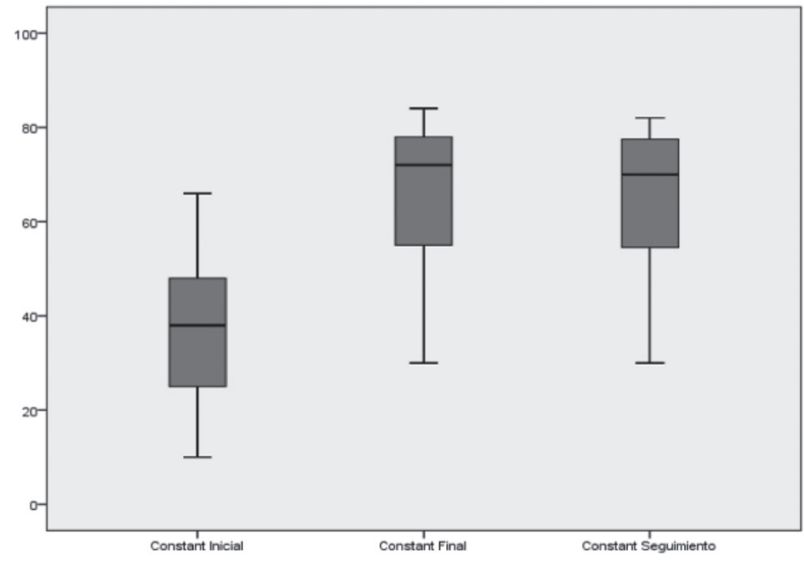

Fig. 6. Puntuación funcionalidad de hombro con score de Constant, preintervención, a la sexta semana y al sexto mes de seguimiento.

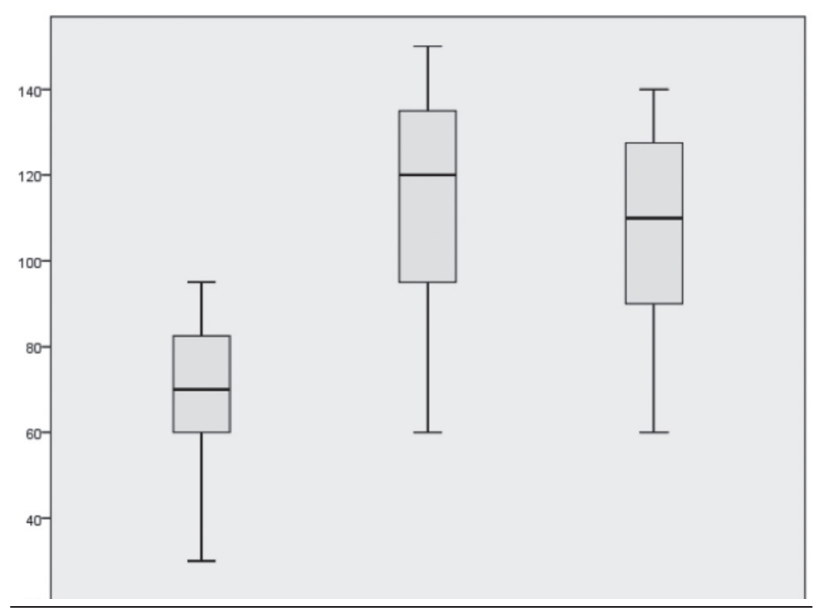

Fig. 7. AROM de flexión de hombro, preintervención, a la sexta semana y al sexto mes de seguimiento.

\section{AROM de abducción}

En el AROM de abducción antes de la intervención el promedio fue de $57,43^{\circ} \mathrm{DE}\left(15,84^{\circ}\right)$. A la sexta semana fue de $90,12^{\circ} \mathrm{DE}\left(28,64^{\circ}\right)$ y al sexto mes fue de $86,92^{\circ} \mathrm{DE}\left(26,09^{\circ}\right)$. Existen diferencias estadísticamente significativas entre el AROM de abducción preintervención versus postintervención $(\mathrm{p}=0,01)$, y además entre el AROM de abducción preintervención versus al final del $6^{\circ}$ mes $(\mathrm{p}=0,02)$ (Figura 8).

\section{DISCUSIÓN}

Se intentó describir los cambios en la función de hombro, en la intensidad de dolor y en los AROM de flexión y

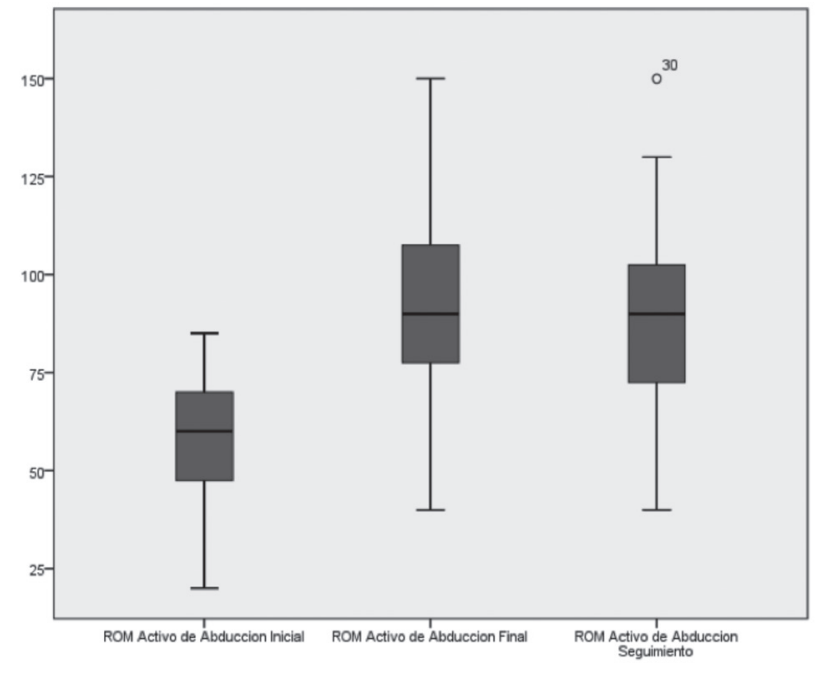

Fig. 8. AROM de abducción de hombro, preintervención, a la sexta semana y al sexto mes de seguimiento.

abducción de hombro, posterior a la intervención de ejercicios de activación selectiva glenohumerales e IMG, a las seis semanas de tratamiento y al sexto mes de seguimiento, en una muestra de 50 sujetos con diagnóstico médico e imagenológico de rotura masiva del manguito rotador.

Los cambios más significativos se observaron en flexión activa de hombro con una mejoría promedio de $40^{\circ}$ a la sexta semana, y $34,87^{\circ}$ al sexto mes, que supera el cambio mínimo detectable para esta variable (32). Con respecto a la función de hombro, se observó una mejoría promedio de 25,43 puntos a la sexta semana y 24,38 puntos al sexto mes. Nuestros resultados son concordantes con estudios previos (35-38) en donde se menciona que la terapia de juicio de lateralidad y la imaginería de movimiento pueden ser consideradas como una forma y/o herramienta de rehabilitación en pacientes con dolor crónico y con alteraciones en el patrón normal de movimiento.

En los últimos años, la inclusión de los enfoques de tratamiento en neurociencia para pacientes con dolor musculoesquelético crónico ha generado un debate dentro de los clínicos, debido a que esta propuesta terapéutica se basa en la integración del sistema nervioso central, como la piedra angular de la rehabilitación, cuestionando los modelos clásicos de intervención que hacen referencia a los mecanismos nociceptivos secundarios a un daño estructural, considerando solo el componente biológico y mecánico $(39,40)$. Algunos estudios han demostrado que el enfoque basado en la neurociencia, como la IMG, ha reportado mejoras en el dolor en sujetos con dolor crónico (41), dolor inespecífico de hombro (27), artritis reumatoide (42) y columna lumbar (24). Asimismo, varios estudios han demostrado que la IMG puede ayudar a la neuroplasticidad cortical generada por el dolor crónico 
$(22,23,37,38,42,43)$. Sin embargo, no hay estudios que consideren estas dos intervenciones por sí solas en los cambios de las variables estudiadas. Esto sustenta que este tipo de intervenciones integra el SNC, haciendo entender todas las alteraciones en el dolor crónico músculo-esquelético y que nuestras estrategias de tratamiento deben ir orientadas a integrar las estructuras cerebrales, y que el SNC sea la puerta de entrada para los tratamientos en estas condiciones clínicas (40).

Con respecto a los ejercicios de activación selectiva, una de las características importantes es que son isométricos a baja carga. Esto repercute directamente desde el punto de vista biomecánico, ya que disminuye la sobrecarga de los tejidos en la articulación, logrando minimizar la demanda mecánica y así evitar el desarrollo de la sintomatología clínica (44-46). Por lo tanto, con estos ejercicios selectivos se lograría trabajar libre de dolor y activando de manera inocua los músculos del manguito rotador, redistribuyendo su actividad muscular $(47,48)$. Por otra parte, la aplicación clínica del ejercicio de depresión humeral se fundamenta biomecánicamente por el ascenso de la cabeza humeral producto del torque del deltoides, ya que un desgarro masivo del músculo supraespinoso, subescapular o infraespinoso genera una sobreactividad del dorsal ancho y pectoral mayor para modular y controlar el ascenso de la cabeza del humero $(7,8)$. Por lo tanto, uno de los objetivos importantes es realizar ejercicios que desciendan la cabeza humeral, distribuyendo su actividad de estos músculos mencionados anteriormente. El ejercicio de activación selectiva del deltoides medio se fundamenta por el signo de pseudoparálisis en esta condición clínica, debido al desgarro del músculo supraespinoso y subescapular. Esto genera una importante limitación de movimientos de elevación de brazo (6). Sin embargo, al activar el músculo deltoides sobre los $45^{\circ}$ de elevación en el plano escapular genera una disminución del área de contactosubacromial y la presión mecánica disminuye significativamente $(6,9)$.

La gran mayoría de los tratamientos conservadores asociados a esta condición clínica están basados en ejercicio, terapia manual, taping, inyección de corticoides y electroterapia (49-51), con un cuestionado éxito terapéutico. Por lo tanto, se hace necesario para la práctica clínica abrir áreas en la rehabilitación motora músculo-esquelética. Desde este punto, en el último tiempo la IMG se ha abierto paso a la rehabilitación músculo-esquelética, demostrando su efectividad $(14,17,24,27)$.

Con respecto a la IMG, algunos estudios de neuroimagen han demostrado que posterior a los entrenamientos con esta herramienta terapéutica, se activa significativamente la corteza premotora durante los ejercicios de reconocimiento de lateralidad $(14,52,53)$. Por lo tanto, estos reportes corroboran que esta herramienta va orientada a revertir cambios a nivel cortical y es una estrategia que abarca el SNC para la rehabilitación motora.

\section{Limitaciones}

Por último, con relación al tipo de estudio, es importante mencionar que este tipo de diseños no permiten realizar una inferencia hacia la población debido a una serie de factores metodológicos. Primero, es un estudio no experimental, por lo tanto no posee un grupo control comparativo para establecer efectividad de una intervención, ni tampoco se seleccionan los sujetos por un muestreo aleatorio probabilístico. Por lo tanto, este tipo de diseños descriptivos solo buscan describir un posible efecto en una variable. Por lo anteriormente mencionado, los resultados de nuestra investigación deben ser interpretados con precaución y utilizados para futuras investigaciones experimentales. Otro aspecto a considerar es la heterogeneidad de la muestra en relación al género, ya que un $36 \%$ eran hombres (18 sujetos) y un $64 \%$ mujeres (32 sujetos), por lo tanto se sugiere que los resultados deben ser tomados con precaución para la posterior aplicabilidad.

\section{CONCLUSIÓN}

Los resultados sugieren que adicionar una intervención de ejercicios de lateralidad e imaginería del movimiento a un programa de ejercicios de activación selectiva de los estabilizadores glenohumerales durante 6 semanas podría mejorar la función de hombro, disminuir el dolor, y aumentar los AROM de flexión y abducción de hombro en pacientes con ruptura masiva del manguito rotador.

\section{CONFLICTO DE INTERESES}

Los autores declaran no tener ningún conflicto de intereses.

\section{BIBLIOGRAFÍA}

1. Boileau P, Brassart N, Watkinson DJ, Carles M, Hatzidakis AM, Krishnan SG. Arthroscopic repair of full-thickness tears of supraspinatus: does the tendon really heal? J Bone Joint Surg Am 2005;87(6):1229-40.

2. Yamamoto A, Takagishi K, Osawa T, Yanagawa T, Nakajima D, Shitara H, et al. Prevalence and risk factors of a rotator cuff tear in the general population. J Shoulder. Elbow Surg 2010;19(1):116-20. DOI: 10.1016/j.jse.2009.04.006.

3. Moosmayer S, Tariq E, Stiris M, Smith HJ. History of asymptomatic rotator cuff tears. Three year follow-up of fifty cases. J Bone Joint Surg Am 2013;95(14):1249-55. DOI: 10.2106/JBJS.L.00185.

4. Denard PJ, Koo SS Murena L, Burkhart SS. Pseudoparalysis: The importance of rotator cable integrity. Orthopedics 2012;35(9):1353-7. DOI: 10.3928/0147744720120822-21.

5. Tokish JM, Alexander TC, Kissenberth MJ, Hawkins RJ. Pseudoparalysis: a systematic review of term definitions, 
treatment approaches, and outcomes of management techniques. J Shoulder Elbow Surg 2017;26(6):177-87. DOI: 10.1016/j.jse.2017.02.024.

6. Cheung S, Dillon E, Tham S, Feeley B, Link TM, Steinbach $\mathrm{L}$, et al. The presence of fatty infiltration in the infraspinatus: its relation with the condition of the supraspinatus tendon. Arthroscopy 2011;27(4):463-70. DOI: 10.1016/j. arthro.2010.09.014.

7. Campbell ST, Ecklund KJ, Chu EH, McGarry MH, Gupta $\mathrm{R}$, Lee TQ. Role of pectoral major and latissimus dorsi in a biomechanical model of massive rotator cuff tear. J Shoulder Elbow Surg 2014;23(8):1136-42. DOI: 10.1016/j.jse.2013.11.030.

8. Park SY, Yoo WG. Selective activation of the latissimus dorsi and inferior trapezius during isometric pull-down exertion. J Electromyogr Kinesiol 2013;23(6):1350-5. DOI: 10.1016/j.jelekin.2013.08.006.

9. Hansen ML, Otis JC, Johnson JS, Cordasco FA, Craig EV, Warren RF. Biomechanics of massive rotator cuff tears. Implications of treatment. J Bone Joint Surg Am 2008;90(2):316-25. DOI: 10.2106/JBJS.F.00880.

10. Hawkes D, Alizadehkhaiyat O, Kemp G, Fisher A, Roebuc M, Frostick FP. Shoulder muscle activation and coordination in patients with a massive rotator cuff tear: An electromyographic study. J Orthop Res 2012;30(7):1140-6. DOI: $10.1002 /$ jor. 22051.

11. Snodgrass SJ, Heneghan NR, Tsao H, Stanwell PT, Rivett DA, Van Vliet PM. Recognising neuroplasticity in musculoskeletal rehabilitation: a basis for greater collaboration between musculoskeletal and neurological physiotherapists. Man Ther 2014;19(6):614-7.

12. Muratori LM, Lamberg EM, Quinn L, Duff SV. Applying principles of motor learning and control to upper extremity rehabilitation. J Hand Ther 2013;26(2):94-102. DOI: 10.1016/j.jht.2012.12.007.

13. Bachasson D, Singh A, Shah S, Lane S, Ward R. The role of the peripheral and central nervous system in rotator cuff disease. J Shoulder Elbow Surg 2015;24(8):1322-35. DOI: 10.1016/j.jse.2015.04.004.

14. Moseley GL. Graded motor imagery is effective for longstanding complex regional pain syndrome: a randomised controlled trial. Pain 2004;108(1-2):192-8.

15. Méndez-Rebolledo G, Gatica-Rojas V, Torres-Cueco R, Albornoz-Verdugo M, Guzmán-Muñoz E. Update on the effects of graded motor imagery and mirror therapy on complex regional painsyndrome type 1: A systematic review. J Back Musculoskelet Rehabil 2017;30(3):441-9. DOI: 10.3233/BMR-150500.

16. Daly AE, Bialocerkowski AE. Does evidence support physiotherapy management of adult Complex Regional Pain Syndrome Type One? A systematic review. Eur J Pain 2009;13(4):339-53.

17. Bowering KJ, O'Connell NE, Tabor A, Catley MJ, Leake $\mathrm{HB}$, Moseley GL, et al. The effects of graded motor imagery and its components on chronic pain: a systematic review and metaanalysis. J Pain 2013;14(1):3-13. DOI: 10.1016/j. jpain.2012.09.007

18. Wand BM, Parkitny L, O'Connell NE, Luomajoki H, McAuley JH, Thacker M, et al. Cortical changes in chronic low back pain: current state of the art and implications for clinical practice. Man Ther 2011;16(1):15-20. DOI: 10.1016/j.math.2010.06.008.

19. Flor $\mathrm{H}$. The functional organization of the brain in chronic pain. In: Sandkühler J, Bromm B, Gebhart GF, eds. Progress in Brain Research: 129. Amsterdam: Elsevier; 2000.
20. Stavrinou ML, Della Penna S, Pizzella V, Torquati K, Cianflone F, Franciotti R, et al. Temporal dynamics of plastic changes in human primary somatosensory cortex after finger webbing. Cereb Cortex 2007;17(9):2134-42.

21. Flor H, Braun C, Elbert T, Birmbaumer N. Extensive reorganisation of primary somato sensory cortex in chronic back pain patients. NeurosciLett 1997;224(1):5-8.

22. Moseley GL, Gallace A, Spence C. Bodily illusions in health and disease: Physiological and clinical perspectives and the concept of a cortical 'body matrix. Neurosci Biobehav Rev 2012;36(1):34-46. DOI: 10.1016/j.neubiorev.2011.03.013.

23. Moseley GL, Butler DS, Beames TB, Giles TJ. The Graded Motor Imagery Handbook. Australia: Adelaide Noi group Publications; 2012.

24. Louw A, Schmidt SG, Louw C, Puentedura EJ. Moving without moving: immediate management following lumbar spine surgery using a graded motor imagery approach: a case report. Physiother Theory Pract 2015;31(7):509-17. DOI: 10.3109/09593985.2015.1060656.

25. Moseley GL. Graded motor imagery for pathologic pain. Neurology 2006;67(12):1-6.

26. McCabe CS, Haigh RC, Ring EF, Halligan P, Blake DR. A controlled pilot study of the utility of mirror visual feedback in the treatment of complex regional pain syndrome (type 1). Rheumatology (Oxford) 2003;42(1):97-101.

27. Louw A, Puentedura EJ, Reese D, Parker P, Miller T, Mintken P. Immediate effects of mirror therapy in patients with shoulder pain and decreased range of motion. Arch Phys Med Rehabil 2017;98(10):1941-7. DOI: 10.1016/j. apmr.2017.03.031.

28. Foell J, Bekrater-Bodmann R, Diers M, Flor H. Mirror therapy for phantom limb pain: brain changes and the role of body representation. European Journal of Pain. May 2014;18(5):729-9. DOI: $10.1002 /$ j.15322149.2013.00433.x.

29. Pardo C, Muñoz T, Chamorro C. Monitorización del dolor: recomendaciones del grupo de trabajo de analgesia y sedación de la SEMICYUC. Med Intensiva 2006;30(8):379-85.

30. Constant C, Gerber C, Emery R, Sojberg J, Gohlke F, Boileau P. A review of the Constant score: Modifications and guidelines for its use. J Shoulder Elbow Surg 2008;17(2):355-61. DOI: 10.1016/j.jse.2007.06.022.

31. Grassi FA, Tajana MS. The normalization of data in the Constant-Murley score for the shoulder. A study conducted on 563 health subjects. Chir Organi Mov 2003;88(1):65-73.

32. McCormack HM, Horne DJ, Sheather S. Clinical applications of visual analogue scales: a critical review. Psychol Med 1988;18(4):1007-19.

33. Kolber MJ, Hanney WJ. The reliability and concurrent validity of shoulder mobility measurements using a digital inclinometer and goniometer: a technicalreport. Int J Sports Phys Ther 2012;7(3):306-13.

34. Cherchi L, Ciornohac JF, Godet J, Clavert P, Kempf JF. Critica shoulder angle: Measurement reproducibility and correlation with rotator cuff tendon tears. Orthop Traumatol Surg Res 2016;102(5):559-62. DOI: 10.1016/j.otsr.2016.03.017.

35. Wand BM, O'Connell NE, Di Pietro F, Bulsara M. Managing chronic nonspecific low back pain with a sensorimotor retraining approach: exploratory multiple-baseline study of 3 participants. Phys Ther 2011;91(4):535-46. DOI: 10.2522/ ptj.20100150.

36. Parsons LM, Fox PT, Downs JH, Glass T, Hirsch TB, Martin CC, Jet al. Use of implicit motor imagery for 
visual shape discrimination as revealed by PET. Nature 1995;375(6526):54-8.

37. Vingerhoets G, de Lange FP, Vandemaele P, Deblaere K, Achten E. Motor imagery in mental rotation: an FMRI Study. Neuroimage 2002;17(3):1623-33.

38. Zacks J, Rypma B, Gabrieli JDE, Tversky B, Glover GH. Imagined transformations of bodies: an fMRI investigation. Neuropsychologia 1999;37(9):1029-40.

39. LluchGirbes E, Meeus M, Baert I, Nijs J. Balancing "handson" with "hands-off" physical therapy interventions for the treatment of central sensitization pain in osteoarthritis. Man Ther 2015;20(2):349-52. DOI: 10.1016/j.math.2014.07.017.

40. Nijs J, Van Houdenhove B, Oostendorp RA. Recognitionof central sensitization in patients with musculoskeletal pain: application of pain neurophysiology in manual therapypractice. Man Ther 2010;15(2):135-41. DOI: 10.1016/j. math.2009.12.001.

41. Louw A, Zimney K, Puentedura EJ, Diener I. The efficacy of pain neuroscience education on musculoskeletal pain: A systematic review of the literature. Physiother Theory Pract 2016;32(5):332-55. DOI: 10.1080/09593985.2016.1194646.

42. Moseley GL, Zalucki N, Birklein F, Marinus J, Van Hilten JJ, Luomajoki H. Thinking about movement hurts: the effect of motor imagery on pain and swelling in people with chronic arm pain arthritis \& rheumatism. Arthritis care \& research. 2008;59(5):623-31. DOI: 10.1002/art.23580.

43. Smart KM, Wand BM, O'Connell NE. Physiotherapy for pain and disability in adults with complex regional pain syndrome (CRPS) types I and II. Cochrane Database Syst Rev 2016; CD010853. DOI: 10.1002/14651858.CD010853.pub2.

44. Walch G, Edwards TB, Boulahia A, Nové-Josserand L, NeytonL, Szabo I. Arthroscopic tenotomy of the long head of the biceps in the treatment of rotator cuff tears: clinical and radiographic results of 307 cases. J Shoulder Elbow Surg 2005;14(03):238-46.
45. Smith J, Dahm DL, Kaufman KR, Boon AJ, Laskowski ER, Kotajarvi BR, et al. Electromyographic activity in the immobilized shoulder girdle musculature during scapulo thoracic exercises. Arch Phys Med Rehabil 2006;87(7):923-7.

46. Uhl TL, Muir TA, Lawson L. Electromyographical assessment of passive, active assistive, and active shoulde rrehabilitation exercises. PM R 2010;2(2):132-1. DOI: 10.1016/j.pmrj.2010.01.002.

47. Koh KH, Kang KC, Lim TK, Shon MS, Yoo JC. Prospective randomized clinical trial of single- versus double-row suture anchor repair in 2- to 4-cm rotator cuff tears: clinical and magnetic resonance imaging results. Arthroscopy 2011;27(4):453-62. DOI: 10.1016/j.arthro.2010.11.059.

48. Alenabi T, Jackson M, Tétreault P, Begon M. Electromyographic activity in the immobilized shoulder musculature during ipsilateral elbow, wrist, and finger movements while wearing a shoulder orthosis. J ShoulderElbowSurg 2013;22(10):1400-7. DOI: 10.1016/j.jse.2013.04.007.

49. Gerber C, Fuchs B, Hodler J. The results of repair of massive tears of the rotator cuff. J Bone Joint Surg Am 2000;82(4):505-15.

50. Krischak G, Gebhard F, Reichel H, Friemert B, Schneider F, Fisser C, et al. A prospective randomized controlled trial comparing occupational therapy with home-based exercises in conservative treatment of rotator cuff tears. J Shoulder Elbow Surg. 2013;22(9):1173-9. DOI: 10.1016/j. jse.2013.01.008.

51. Page MJ, Green S, McBain B, Surace SJ, Deitch J, Lyttle N, et al. Manual therapy and exercise for rotator cuff disease. Cochrane Database Syst Rev 2016;(6):CD012224. DOI: 10.1002/14651858.CD012224.

52. Parsons LM. Integrating cognitive psychology, neurology and neuroimaging. Acta Psychol 2001;107(1-3):155-81.

53. Decety J. Do imagined and executed actions share the same neural substrate? CognitBrain Res 1996;3(2):87-93. 